\title{
Interaction between the RF Field of MRI Apparatus and Pacemakers
}

\author{
Stefano Pisa, Paolo Bernardi, and Emanuele Piuzzi \\ Sapienza University of Rome \\ Italy
}

\section{Introduction}

Magnetic resonance imaging (MRI) is a tomography technique that measures the radio frequency (RF) field produced by the magnetic moments of hydrogen nuclei during their precession following the application of RF pulses, superimposed to a static magnetic field.

MRI is a widely accepted tool for the diagnosis of a variety of diseases. Nowadays, however, MRI is contraindicated for patients implanted with pacemakers (PM) due to possible adverse effects (ICNIRP, 2004). The most important of such effects seems to be the heating of the heart tissue around the catheter tip resulting from the high currents induced on the catheter by the RF field used in MRI technique (Nyenhuis, 2005).

In order to avoid thermal hazards resulting from MRI investigations, international agencies have issued guidelines reporting recommended limits. The International Commission on NonIonizing Radiation Protection (ICNIRP, 2004) considers that for whole-body exposures to MRI apparatus, no adverse health effects are expected if the increase in body core temperature does not exceed $1{ }^{\circ} \mathrm{C}$. With regard to localized heating, ICNIRP assumes that adverse effects are avoided with a reasonable certainty if temperature remains lower than $38{ }^{\circ} \mathrm{C}$ in localized regions of the head, lower than $39{ }^{\circ} \mathrm{C}$ in the trunk, and less than $40{ }^{\circ} \mathrm{C}$ in the limbs. Accordingly, in ICNIRP (2004) limitations have been reported on the maximum power, expressed in terms of specific absorption rate (SAR), namely power per unit mass of tissue $(\mathrm{W} / \mathrm{kg})$, that can be dissipated inside the patient body during MRI investigations. More in detail, such limitations refer to whole body SAR $\left(\mathrm{SAR}_{\mathrm{WB}}\right)$ and local SAR as averaged over $10 \mathrm{~g}$ of tissue $\left(\mathrm{SAR}_{10}\right)$. In particular, in normal conditions, the $S A R_{W B}$ should not exceed $2 \mathrm{~W} / \mathrm{kg}$, while the $\mathrm{SAR}_{10}$ is limited to $20 \mathrm{~W} / \mathrm{kg}$ in the extremities and $10 \mathrm{~W} / \mathrm{kg}$ in the head and trunk.

The above reported SAR limitations should ensure safe MRI investigations for normal patients, but might not guarantee adequate protection for PM holders, mainly because of the previously mentioned catheter tip heating (Bernardi et al., 2009). To clarify this issue, the amount of heating in PM holders during MRI sessions has been investigated in several in vivo, in vitro, and numerical studies and reported temperature elevations spread from not significant values up to tens of degrees. Hence, in this Chapter, the studies available in the literature and concerning SAR distributions and temperature increments in PM holders, exposed to the field generated by MRI apparatus, will be described in order to evidence the main factors influencing the obtained SAR values and temperature increments. After a preliminary description of MRI apparatus in terms of field sources and distributions, the main in vitro (on phantoms), and numerical literature results will be presented. In 
particular, the numerical literature survey will show that some authors used accurate models of the pacemaker, embedded in coarse body models, while other authors used anatomically correct body models, with crude pacemakers realizations. Both these approaches can overestimate or underestimate the actual temperature increases in pacemaker holders under MRI investigations. Hence, in the final part of this chapter, SAR distributions and temperature increments in PM holders, exposed inside an MRI apparatus, will be evaluated by using an anatomically based human body phantom together with fine pacemaker models equipped with different kinds of catheters, implanted following the vein path. This study will be performed by using a conformal FDTD code with graded mesh (CGM-FDTD) for the solution of the electromagnetic (EM) problem, and a finite difference solution of the bioheat equation with graded mesh, for the evaluation of the corresponding temperature increments. This model will allow to investigate the influence of the PM and antenna geometry on the induced thermal increments, and to give some rules for the design of antenna or catheter geometries able to make MRI safe for PM holders.

\section{MRI fields and coils}

\subsection{MRI apparatus}

Magnetic resonance imaging technique is based on induced nuclear magnetic resonance of water molecules. For this reason MRI is the imaging technique of choice for pathologies where it is important to discriminate among different soft (well hydrated) tissues like brain, muscle, and tumors. MRI provides good spatial resolution (the ability to distinguish between two separate structures at an arbitrarily small distance from each other), and a high contrast resolution (the ability to distinguish the differences between two similar but not identical tissues). Unlike traditional X-rays and computer tomography (CT), MRI does not use ionizing radiation and it is generally a very safe procedure.

The human body is largely composed of water molecules. Each water molecule has two hydrogen atoms with a proton inside the nucleus. During an MRI examination of a person a static magnetic field $B_{0}$ produced by superconductive magnets $\left(B_{0}\right.$ coil in Fig. 1$)$ aligns the spin magnetic dipole moments of hydrogen protons in the direction of the applied field.

Each proton, or dipole moment, precesses about the static field, at the Larmor frequency $\mathrm{f}_{0}=\gamma \mathrm{B}_{0}$ (where $\gamma \approx 42.58 \mathrm{MHz} /$ tesla). A radio frequency magnetic field that oscillates at $\mathrm{f}_{0}$, is applied using an appropriate coil (RF tx/rx coil in Fig. 1) in the direction transverse to $\mathrm{B}_{0}$ and by choosing the pulse duration of the excitation, the magnetic dipole moments can be rotated into the transverse direction. The decaying signal following the pulse excitation is detected using a RF receiving antenna. Spatially varying magnetic fields are applied during the scan in order to make the magnetic field strength dependent on the position within the patient, providing a straightforward method to control where the protons are excited by the RF field. These varying magnetic fields are generated by passing currents through solenoids, known as gradient coils (see Fig. 1). For an image of high quality, the applied RF magnetic field should be uniform over all regions of the body being imaged since a non uniform field introduces distortion into the image.

Moreover, for maximum energy coupling into the hydrogen nuclei it is necessary to have an antenna that can generate circularly polarized fields. Birdcage and TEM coils have been designed to satisfy both of these requirements and will be briefly described in the following. 


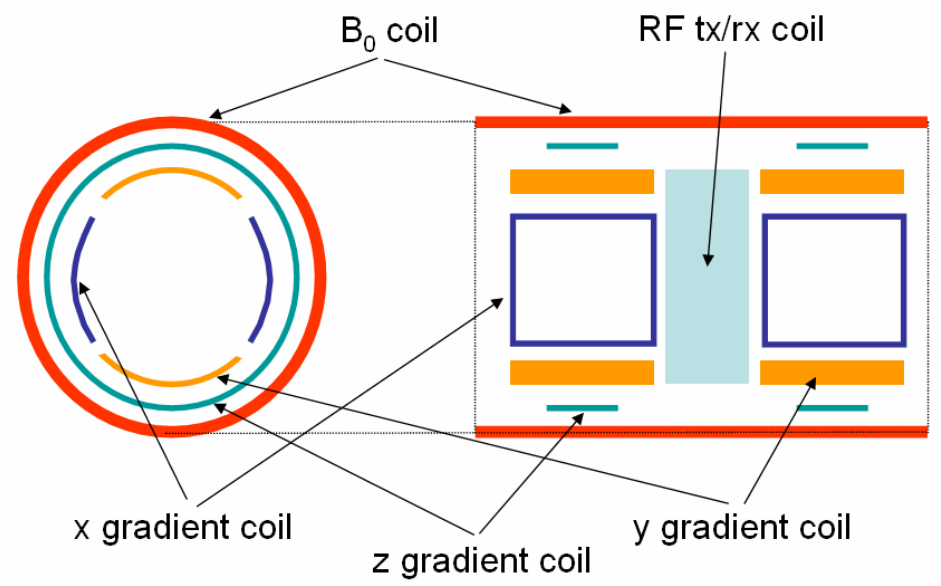

Fig. 1. MRI tomograph composed of five coils: one for the main static field $\mathrm{B}_{0}$; three for the gradients and one for the RF transmission/reception

\subsection{Birdcage coils}

Birdcage coils are constituted by two conducting end-rings (ERs) connected by a number of conducting legs (rungs) distributed at equal spacing around the perimeter of the ERs (Jin, 1999). Capacitors are inserted into legs or ERs to achieve the tuning of the desired resonant mode. A low-pass (LP) coil has capacitors placed in the legs while a high-pass (HP) coil has the capacitors situated in ER segments. Fig. 2 shows a low-pass birdcage coil (a) and a highpass birdcage coil (b) geometry modeled inside the commercial CAD Microwave Studio (MWS).

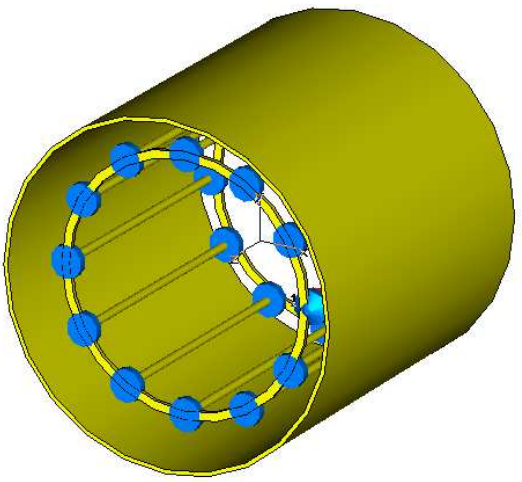

(a)

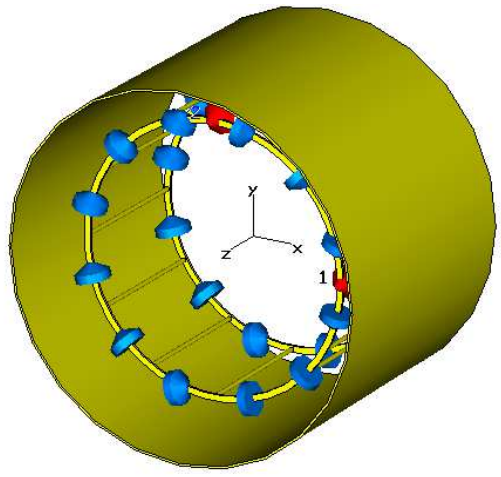

(b)

Fig. 2. Low-pass birdcage coil (a); high-pass birdcage coil (b). Tuning capacitors are evidenced as blue disks

Capacitors are evidenced as blue disks and a cylindrical shield, that prevents the field from being radiated outside the coil, is added in the CAD model. 
Birdcage coils are resonators characterized by more than one resonant mode: in fact, an Nleg coil has (N/2) +1 distinct resonant modes (Jin, 1999). According to the theory reported in Giovannetti et al. (2002), the resonant frequencies for a low-pass birdcage are given by:

$$
\omega_{k}=\left[C\left(\operatorname{Ler}+\operatorname{Lleg} / 2 \sin ^{2} \frac{\pi k}{N}\right)\right]^{-1 / 2} \quad(k=0,1,2, \ldots . N / 2)
$$

Similarly, for a high-pass coil the resonant frequencies are:

$$
\omega_{k}=\left[C\left(\operatorname{Ler}+2 \cdot \operatorname{Lleg} 2 \sin ^{2} \frac{\pi k}{N}\right)\right]^{-1 / 2} \quad(k=0,1,2, \ldots . N / 2)
$$

where Lleg represents the self-inductance of one leg, $C$ the capacitance of the capacitor between two legs and Ler the self-inductance of the end-ring segments.

By applying a $50 \Omega$ source between the leg and the ring (close to a capacitor) in the MWS model of the birdcage the reflection coefficient (S11) of the birdcage as a function of the frequency has been computed. Fig 3. shows the simulated magnitude of S11 for the low-pass birdcage coil $(\mathrm{N}=12)$ shown in Fig. 2a. The seven resonances of the structure are evidenced in the figure as negative peaks in the spectrum. For the considered birdcage geometry the figure evidences a first resonance at about $128 \mathrm{MHz}\left(\mathrm{B}_{0}=3 \mathrm{~T}\right)$.

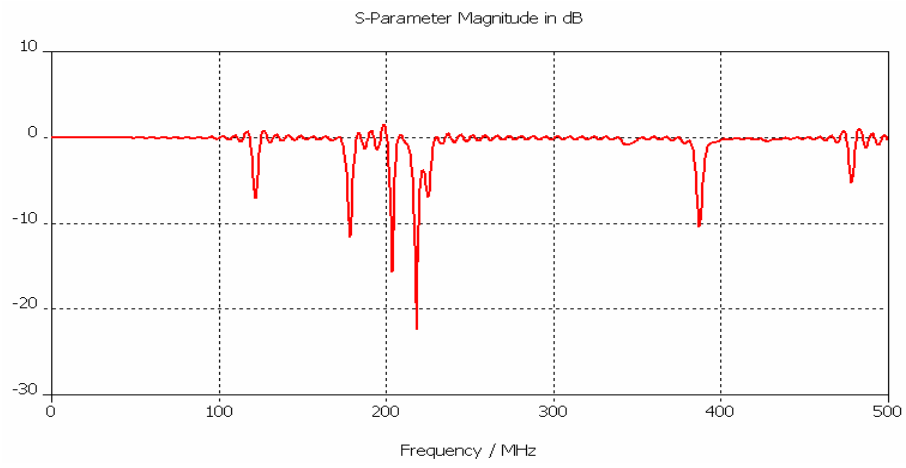

Fig. 3. Simulated $\mathrm{S} 11$ magnitude spectrum for the low-pass birdcage coil $(\mathrm{N}=12)$ shown in Fig. 2a

For a birdcage coil with $\mathrm{N}$ legs, the current can be supposed to be constant along the legs with a co-sinusoidal amplitude variation among legs given by: $\mathrm{I}_{\mathrm{k}}=\cos 2 \pi \mathrm{k} / \mathrm{N}$ where $\mathrm{k}=0,1,2, \ldots \mathrm{N} / 2$ is the mode order. Using the Biot-Savart equation, the magnetic field distribution can be computed from conductor currents associated with a given mode sustained by the birdcage. Note that the use of Biot-Savart equation implies a quasi-static magnetic field assumption, and it is valid when the coil dimensions are much smaller than the wavelength. For example, at $64 \mathrm{MHz}\left(\mathrm{B}_{0}=1.5 \mathrm{~T}\right)$, the wavelength is $4.7 \mathrm{~m}$; thus the quasistatic assumption holds for practical birdcage dimensions. This approximation also holds when increasing the static magnetic field strength, if the coil dimension decreases.

By using MWS the magnetic field inside the birdcage obtained by applying two excitation with $90^{\circ}$ time phase shift in correspondence of two legs with $90^{\circ}$ spatial shift has been 
computed. This kind of excitation simulates the one usually adopted in real birdcages and produces a magnetic field with a circular clockwise polarization, with respect to the positive z-axis (see Fig. 2). A map of the computed magnetic field at $128 \mathrm{MHz}$ (first resonance from the left in Fig. 3) on an axial (a) and a transversal (coronal) (b) section is reported in Fig. 4. The figure evidences a good uniformity of the field in the central region of the birdcage with the highest field values obtained in the region between the leg and the shield.

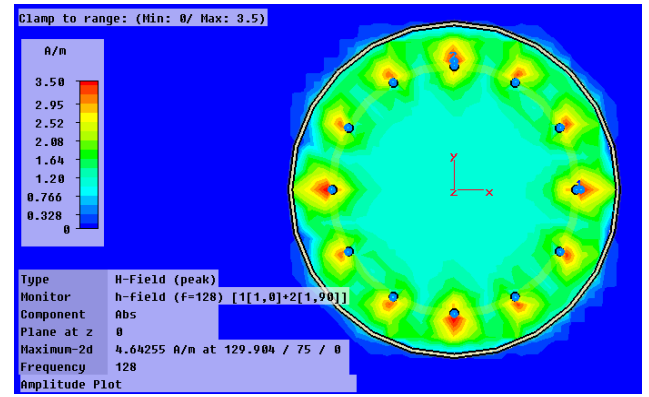

(a)

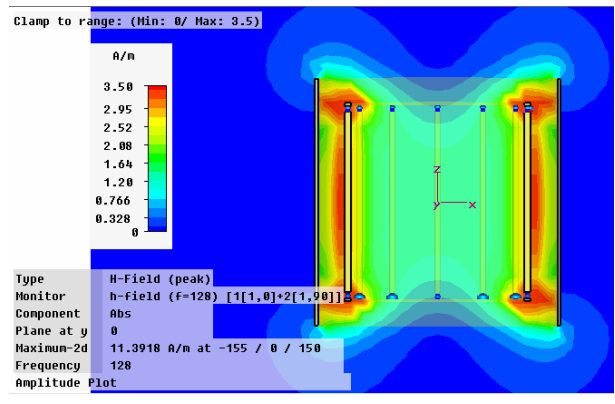

(b)

Fig. 4. Maps of the computed magnetic field at $128 \mathrm{MHz}$ inside the birdcage on axial (a) and transversal (coronal) $(b)$ sections

\subsection{TEM coils}

The needs of using higher static magnetic fields $\mathrm{B}_{0}$, and, consequently, higher Larmor frequencies for obtaining better MR images has introduced systems operating at $3-9.4 \mathrm{~T}$ $(128-400 \mathrm{MHz})$. This development requires new types of antennas. One of the most commonly used is the transverse electromagnetic (TEM) coil proposed by Röschmann (1988) and Bridges (1988). These coils are based on TEM transmission lines with capacitive loads of various configurations, and have become known as "TEM resonators" (Röschmann, 1987).

The geometries of two TEM resonators are shown in Fig. 5. These resonators consist of multiple longitudinal conductors arranged in a circular cylindrical pattern and enclosed by a cylindrical shield with end-plates that form a cavity. Fig. 5 shows the most commonly used designs in which the inner conductors are metallic tubes (a) or strip conductors over a dielectric (b). The inner conductors connect to the end plates by tuning capacitors (blue disks in Fig. 5). These coils are driven by sources connected to the end of one or more conductors. Theoretical studies on TEM resonators have shown that they behave like loaded multiconductor transmission lines capable of supporting standing waves (Bogdanov \& Ludwig 2002).

In the absence of excitation, and for symmetrically placed tubes, the condition for resonance results in:

$$
\prod_{n=1}^{N}\left(1-\Gamma_{n}^{+} \Gamma_{n}^{-} e^{-2 j k_{n} l}\right)=0
$$

where $\Gamma_{n}^{+}$and $\Gamma_{n}^{-}$are the modal decompositions of the reflection coefficients at the end of the lines and $k_{n}$ is the wave number of mode $n$, which for TEM modes and lossless conductors is given by: $k_{n}=\omega_{n} / c$. The frequency response of the $\mathrm{N}=12$ element coil in Fig. 
5a has been simulated by using MWS. The obtained return loss (S11 magnitude) at the input of this coil is shown in Fig. 6 . It can be seen that N/2 +1 = 7 modes are present.

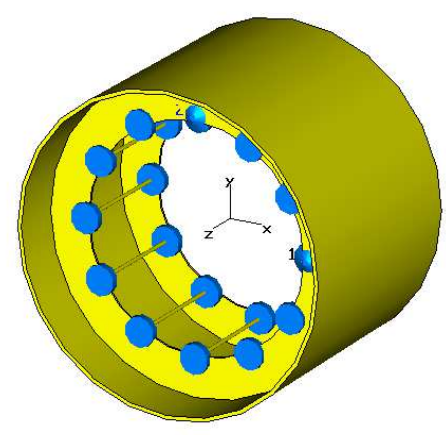

(a)

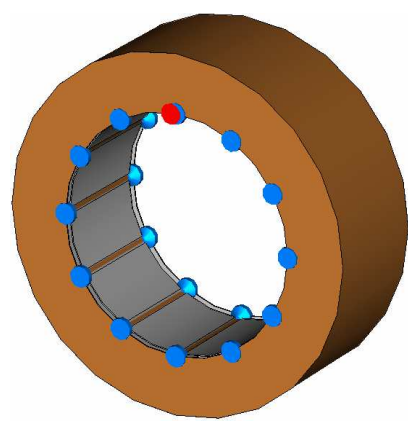

(b)

Fig. 5. TEM resonators: structure in which the inner conductors are metallic tubes (a) or strip conductors over a dielectric (b). Capacitors are evidenced as blue disks.

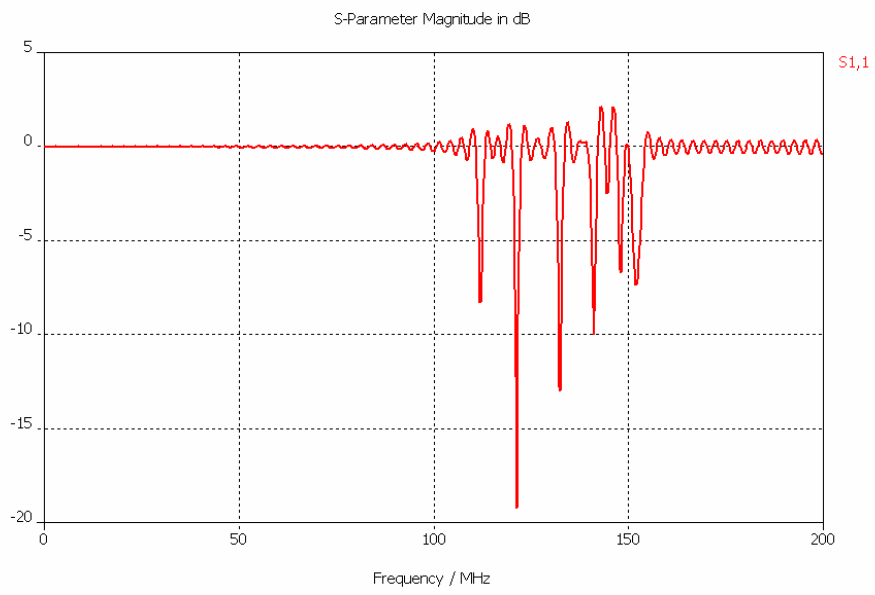

Fig. 6. Simulated S11 magnitude spectrum for the TEM resonator $(\mathrm{N}=12)$ shown in Fig. 5a The magnetic field distribution inside the coil can be determined by the conductor currents according to:

$$
\underline{\mathbf{H}}=\sum_{n}\left[I_{n}^{m+} \underline{\underline{e^{-j k_{n} z}}}-I_{n}^{m-} \underline{\underline{e^{+j k_{n} z}}}\right] \underline{\mathbf{h}}_{n}(x, y)
$$

where $\underline{\mathbf{h}}_{\mathrm{n}}$ is the transverse vector mode function (Bogdanov \& Ludwig 2002).

Because circular polarization is necessary to excite the MR signal, coils are commonly driven in quadrature so as to produce circularly-polarized fields. Fig. 7 shows a map of the magnetic field at $128 \mathrm{MHz}$ (second resonance from the left in Fig. 6) computed by using MWS and the model reported in Fig. 5.a. 


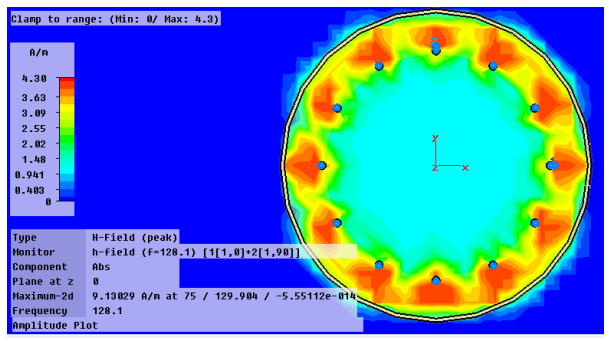

(a)

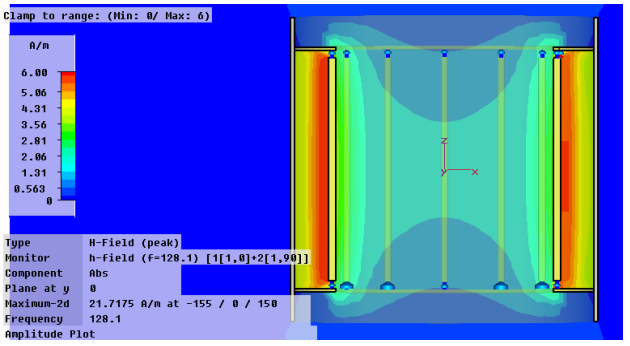

(b)

Fig. 7. Maps of the computed magnetic field at $128 \mathrm{MHz}$ inside the TEM coil on axial (a) and transversal (coronal) (b) sections

In particular, the figure shows maps on axial (a) and transversal (coronal) (b) sections. Similarly to the birdcage the figure evidences a good uniformity of the field in the central region, with the highest field values obtained in the region between the leg and the shield.

\section{In vitro studies}

In vitro studies are usually performed by using commercial MRI apparatus or dedicated experimental setups as the one shown in Fig. 8.

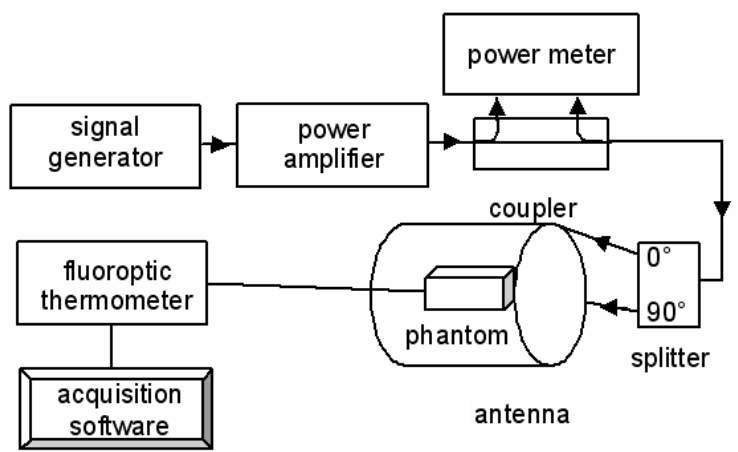

Fig. 8. Experimental setup

In the dedicated system of Fig. 8, the $64 \mathrm{MHz}$ signal is first amplified and then sent to the antenna through a power splitter with $90^{\circ}$ output shift. The applied RF power is monitored with a power meter. When commercial MRI systems are used, the pulse sequences used during regular clinical MR protocol are applied. The whole body SARs in a $80 \mathrm{~kg}$ body produced by the applied pulse levels and width are usually reported on the control display of the system.

Various human body models have been considered. During the experiments both with commercial MRI apparatus and dedicated experimental setup they are inserted inside the system antenna. The simplest phantom is constituted by a parallelepiped box (i.e $30 \mathrm{~cm} \times 20$ $\mathrm{cm} \times 60 \mathrm{~cm}$ ) filled with gelled saline material (i.e. $\mathrm{HEC} 2 \%, \mathrm{NaCl} 0.36 \%$ ) that mimics the electrical and thermal properties of an average human tissue at the considered frequency 
(for example at $64 \mathrm{MHz}: \varepsilon_{\mathrm{r}}=78.2, \sigma=0.6 \mathrm{~S} / \mathrm{m}, \mathrm{K}=0.2 \mathrm{~W} /\left(\mathrm{m}{ }^{\circ} \mathrm{C}\right), \mathrm{C}=4178 \mathrm{~J} /\left(\mathrm{kg}{ }^{\circ} \mathrm{C}\right)$ ) (Bassen et al., 2006; Pisa et al., 2008). A little bit more realistic is the phantom proposed by the American Society for Testing and Materials (ASTM) (ASTM 2007) in which a box model of the thorax $(43.2 \mathrm{~cm} \times 9 \mathrm{~cm} \times 61 \mathrm{~cm})$ is considered together with a head model, in the shape of a box $(16.5 \mathrm{~cm} \times 9 \mathrm{~cm} \times 29.2 \mathrm{~cm})$ (see Fig. 9.a).

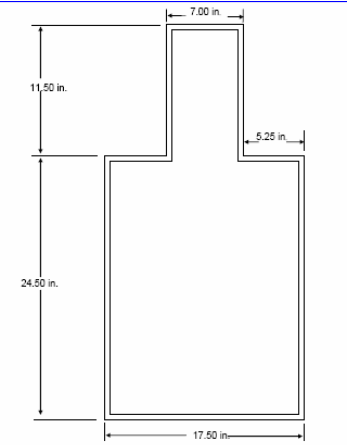

(a)

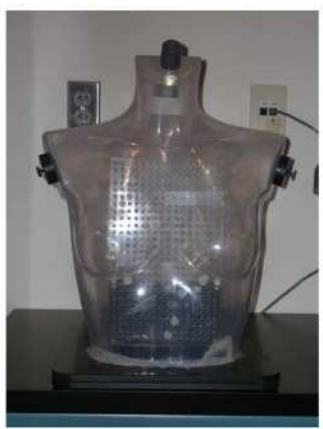

(b)

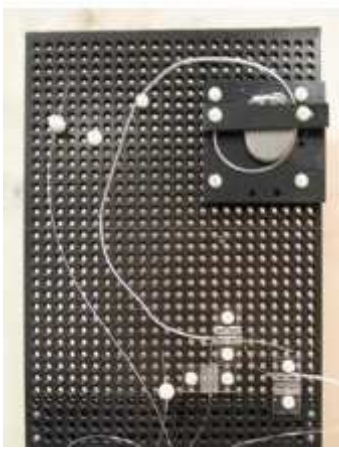

(c)

Fig. 9. ASTM phantom (a); human shaped trunk model (b); plastic grid with a PM (c)

Human shaped trunk models have been also realised by using torso-shaped transparent PVC phantoms (see Fig. 9b) (Mattei et al., 2008). Commercial PM and catheter are fixed to plastic grids (see Fig. 9c) and immersed in the phantom at a depth of about $1 \mathrm{~cm}$ inside the saline material.

Temperature increments are monitored via non perturbing thermometers. As an example, the Luxtron 3100 fluoroptic $^{\mathrm{TM}}$ equipped with SMM probes can be cited. These sensors can reach an accuracy of $0.1^{\circ} \mathrm{C}$ at the point of calibration.

Specific absorption rate (SAR) values are extrapolated from initial temperature rise rate through the equation:

$$
S A R=\left.C \frac{d T}{d t}\right|_{t=0}[\mathrm{~W} / \mathrm{kg}]
$$

In order to reduce the thermal diffusion influence on SAR determination, the temperature variation is usually computed between 3 and $10 \mathrm{~s}$ from the beginning of the exposure. Moreover, the probe positioning close to the catheter must be accurately chosen in order to avoid strong errors in temperature and SAR evaluations (Mattei et al., 2007).

\subsection{Experimental results}

In order to investigate the amount of heating in the heart tissue around the catheter tip in patients implanted with pacemakers many in vitro (on phantom) studies have been performed.

Achenbach et al. (1997) exposed 11 PM inside a commercial MRI scan. For a particular PM lead, they reported a temperature increase of $63.1^{\circ} \mathrm{C}$ after $90 \mathrm{~s}$ of exposure. In other seven electrodes the temperature increase exceeded $15^{\circ} \mathrm{C}$. 
Sommer et al. (2000) exposed 21 PM models with 44 PM electrodes to MRI scanner using a pulse sequence that produces a whole body SAR of $1.3 \mathrm{~W} / \mathrm{kg}$. The PM catheters were inserted into the right ventricle of an isolate porcine heart, connected to a PM case and placed inside a box model of the thorax. The obtained temperature increases ranged from 0.1 to $23.5^{\circ} \mathrm{C}$, depending on the electrode type.

Roguin et al. (2004) applied to PMs placed in a box model of the thorax sequences with SAR less than $1.4 \mathrm{~W} / \mathrm{kg}$ (regular clinical MR protocol) and $3.54 \mathrm{~W} / \mathrm{kg}$ (non clinical protocol). Non clinical protocol gave rise to temperature increments at the catheter tip from 1.5 to 5.7 ${ }^{\circ} \mathrm{C}$, while for clinical protocol the maximal heating was $0.9^{\circ} \mathrm{C}$.

Shellock et al. (2007) tested modern-day (manufactured after year 2000) pacemakers with an experimental set-up like the one shown in Fig. 8 and using the ASTM phantom. Temperature increments were lower than $0.5{ }^{\circ} \mathrm{C}$ for scan of the head and lumbar regions with SAR up to $3 \mathrm{~W} / \mathrm{kg}$. For the chest area temperature increases ranged between 0.4 to 3.6 ${ }^{\circ} \mathrm{C}$ at SAR of $2.0 \mathrm{~W} / \mathrm{kg}$.

Mattei et al., (2008) performed various measurements aimed at the identification of the major contributions involved in the heat generation. In particular they varied the position of the implant, inside box and human shaped phantoms, the length of the wire, the thickness of the insulation sheath, the wire geometries (shape) and the position of the implant including its lead with respect to the RF coil. They found that closer locations of the leads to the edge of the phantom and to the edge of the coil produce maximum heating. The lead length is the other crucial factor, whereas the implant area does not seem to have a major role in the induced temperature increase. Also the lead structure and the geometry of the phantom revealed to be elements that can significantly modify the amount of heating.

\section{Numerical studies}

Numerical studies on the interaction between RF field produced by MRI systems and PM can help in understanding the interaction mechanisms between the field and the PM and can be used to study SAR distributions and temperature increments produced by MRI apparatus inside realistic human body models (Pisa et al., 2010).

\subsection{Human body models}

In numerical studies the body models used in experiments (see Par. 3) can be used. For more realistic evaluations, anatomically heterogeneous body models have been realized. These models consist of large datasets obtained from MRI, CT, and anatomical images. To be used for computational electromagnetic dosimetry these digital data sets must be converted to a so-called "segmented" version. A segmented model is a model where every pixel, usually called in such models "voxel", contains a label which is uniquely associated to a given tissue. In such a way, it is possible to know which tissue fills each of the model voxels and hence assign the correct complex permittivity values to be used in numerical simulations.

In 1988, a high resolution human model has been obtained starting from the "Visible Human Project", developed by the National Library of Medicine (Ackerman, 1998). The Visible human is a 3-D digital image library representing an adult human male and female (see Fig. 10a).

The data sets for both the male and female include photographic images obtained through cryosectioning of human cadavers, and digital images obtained through computer tomography and magnetic resonance imaging of the same cadavers. The segmentation 


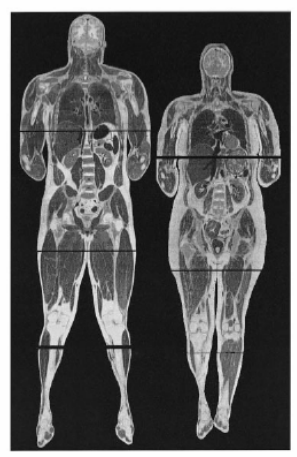

(a)

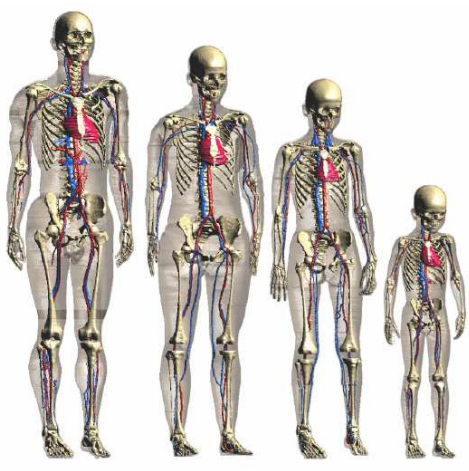

(b)

Fig. 10. Visible human male and female (a); virtual family (b)

procedure has been carried out for the male model by researchers at the Air Force Research Laboratory, Brooks Air Force Base, Texas, USA (Mason et al., 2000). The final segmented model comprises $586 \times 340 \times 1878$ voxels with a resolution of $1 \times 1 \times 1 \mathrm{~mm}^{3}$, and is segmented in about 40 different tissue types.

More recently Christ et al. (2010) have developed four anatomically correct whole body human models: an adult male (34 years old), an adult female (26 years old), and two children (an 11year-old girl and a six-year-old boy) (see Fig. 10b). These four models are referred to as the Virtual Family and are based on high resolution magnetic resonance (MR) images of healthy volunteers. More than 80 different tissue types were distinguished during the segmentation.

\subsection{Catheter models}

Various catheter models have been proposed in literature. The most used is a simple bare cylindrical copper wire (see Fig. 11a) with various diameters and lengths (Ho, 2001; Golombeck \& Dossel, 2004; Stuchly et al., 2006; Pisa et al., 2008), while a more realistic version is constituted by a copper wire covered with plastic (see Fig. 11b). This last catheter

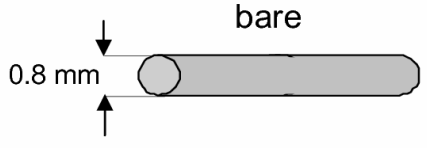

(a)

uncapped catheter

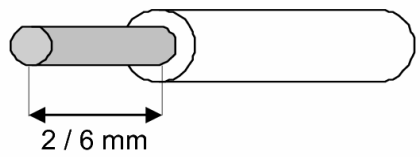

(c)

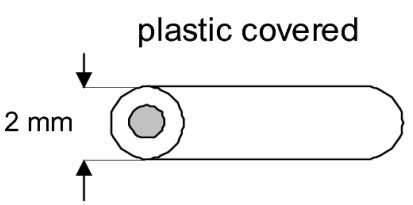

(b)

Helical wire

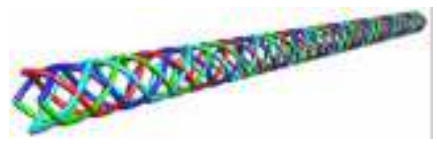

(d)

Fig. 11. Bare (a), plastic covered (b), uncapped (c), and helical wire (d), catheters 
model has been further improved by removing between 2 and $6 \mathrm{~mm}$ of plastic from the tip (uncapped catheters) (see Fig. 11c) (Park et al., 2005; Pisa et al., 2010).

More recently, helical wire models have been proposed (see Fig. 11d) (Bassen et al. 2006; Neufeld et al., 2009). In particular Neufeld et al., (2009) realized their catheter staring from a $0.6 \mathrm{~mm}$ thick wire wound to form a helix and including a $10-\mathrm{mm}$ long straight section. The helical wire is insulated by a thin coating $(0.012 \mathrm{~mm})$.

\subsection{Pacemaker and body placement}

The whole numerical set-up is made by a PM embedded inside a body model in turn inserted inside a coil. The PM is constituted by various lengths of the previously described catheters and by a metallic model of the casing. In the simplified models of the thorax the $\mathrm{PM}$ is inserted at a depth of about $1 \mathrm{~cm}$ inside the phantom following a planar geometry and the phantom is inserted inside the antenna (see Fig. 12a and 12b). When more realistic body models are considered the catheter follows the vein path with a placement approximating the clinical one from the casing to the heart and the anatomical model is inserted inside the coil (see Fig. 12c)

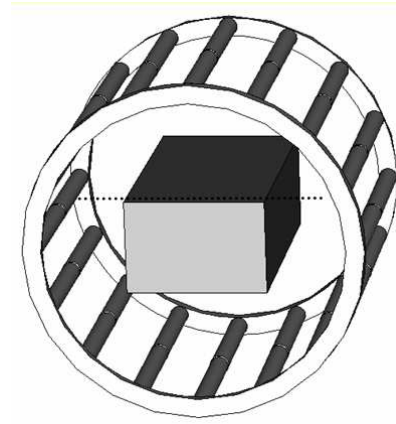

(a)

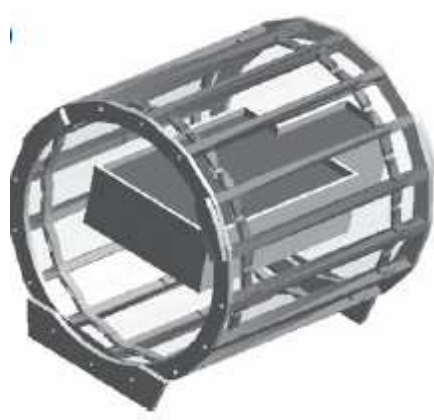

(b)

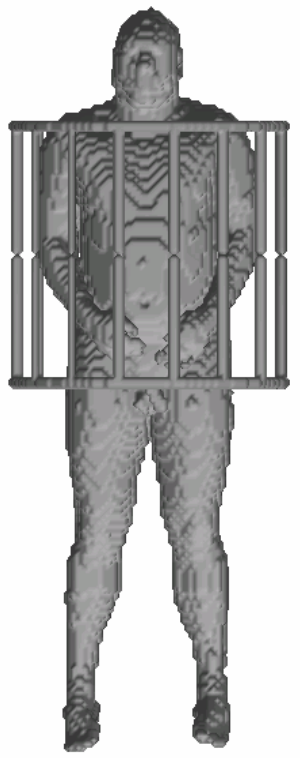

(c)

Fig. 12. Box (a), ASTM (b), and VH models inserted inside RF coils

\subsection{Numerical results}

The above cited human body and PM models have been used to study the interaction of the RF field produced by MRI antennas and pacemaker holders. These studies have been performed by using numerical technique for the solution of the EM and thermal problem, respectively. Among the EM solvers those based on the finite difference time domain (FDTD) technique are the most popular (Taflove \& Hagness, 2000) but also the method of 
moments (MoM) has been largely used (Burke \& Poggio, 1981). For the solution of the thermal problem the Bio-heat equation (Pennes, 1948) has been solved by means of explicit or implicit (ADI) solutions (Bernardi et al., 2003; Pisa et al., 2003). In particular, the method of moments (MoM) (Park et al., 2005; Nyenhuis et al., 2005) and a conformal finite difference time domain (FDTD) method (Pisa et al., 2008) together with a finite difference solution of the bioheat equation have been used for studying the current along the catheter and the temperature distribution around the electrode tip produced by a realistic PM immersed in a homogeneous medium. For bare catheters, the obtained temperature rise ranges from 1 to 28 ${ }^{\circ} \mathrm{C}$ depending on the catheter length and position. Moreover, a commercially available computer aided design (CAD) has been used for evaluating the SAR distribution produced in a realistic human model by a coarse catheter model $(1 \mathrm{~cm}$ diameter, $8 \mathrm{~cm}$ length) implanted in the heart region (Ho, 2001). With this model, a peak SAR averaged over $1 \mathrm{~g}$ of $2 \mathrm{~W} / \mathrm{kg}$ near the metallic implant (compared to the value of $1.8 \mathrm{~W} / \mathrm{kg}$ at the same location without the metallic implant) has been computed and no appreciable heating has been obtained. Similar results have been obtained in Golombeck \& Dossel (2004) and Stuchly et al. (2006).

In order to investigate the effects of the PM geometrical parameters Pisa et al (2008) studied the exposure to a power of $100 \mathrm{~W}$ at $64 \mathrm{MHz}$ of a box model of the thorax in the presence of a pacemaker with a unipolar catheter. A PM with a 60-cm long catheter $\left(l_{1}=10 \mathrm{~cm}, l_{2}=26\right.$ $\mathrm{cm}, l_{3}=10 \mathrm{~cm}, l_{4}=14 \mathrm{~cm}$ in Fig. 13a) was placed inside the thorax. The PM was located in the left side in a typical operating position with the catheter tip $7.5 \mathrm{~cm}$ from the pacemaker.

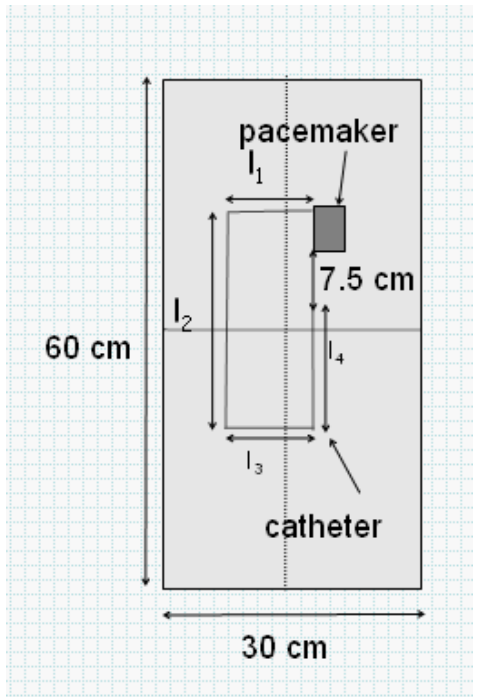

(a)

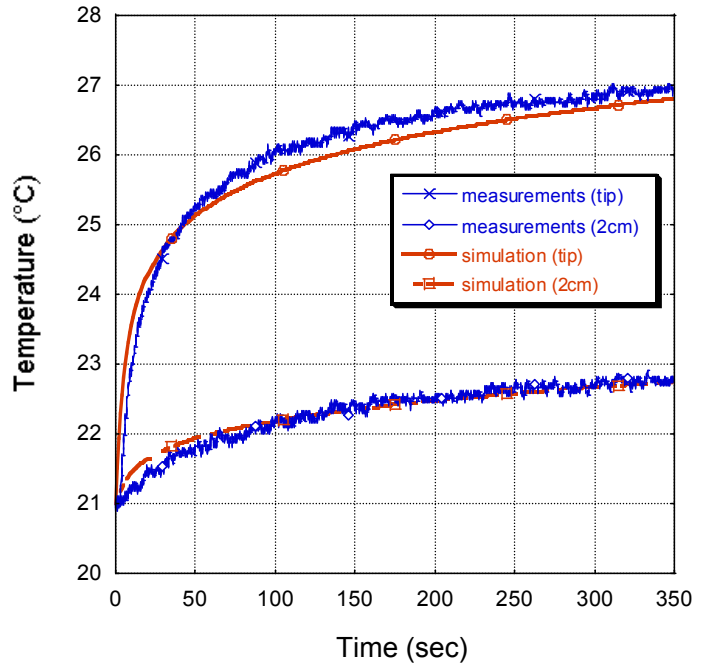

(b)

Fig. 13. Section of the phantom model $1 \mathrm{~cm}$ below the phantom surface showing the geometry of the PM and catheter (a); time behaviour of the temperature on the experimental and numerical phantom at the catheter tip (b)

The exposure has been simulated by using the C-GM-FDTD code for the solution of the EM problem and the ADI-FD code for the solution of Fourier's equation. In the EM simulations 
the PM has been modeled as a copper box $\left(40 \times 10 \times 50 \mathrm{~mm}^{3}\right)$ and the $60-\mathrm{cm}$ long catheter has been modeled has a cylindrical wire of copper with a radius of $0.4 \mathrm{~mm}$. This numerical setup simulates the one adopted in experiments (Pisa et al., 2006). Fig. 13b shows the time behavior of the simulated and measured temperature. After 6-min exposure, a temperature increment of $6^{\circ} \mathrm{C}$ at a point just above the catheter tip and $1.8^{\circ} \mathrm{C}$ at a point $2 \mathrm{~cm}$ below the catheter tip, can be observed, with a good agreement between measurements and simulations. SAR PEAK of $2400 \mathrm{~W} / \mathrm{kg}$ have been obtained at the catheter tip with a SAR $\mathrm{WB}_{\mathrm{B}}$ of about $1.0 \mathrm{~W} / \mathrm{kg}$.

The current distribution along the catheter has been computed as the circulation of the magnetic field around the catheter axis. Fig. 14a shows the obtained current distribution. The distance along the wire from the point in which the catheter is inserted in the pacemaker is reported on the horizontal axis (the catheter length is $60 \mathrm{~cm}$ ).

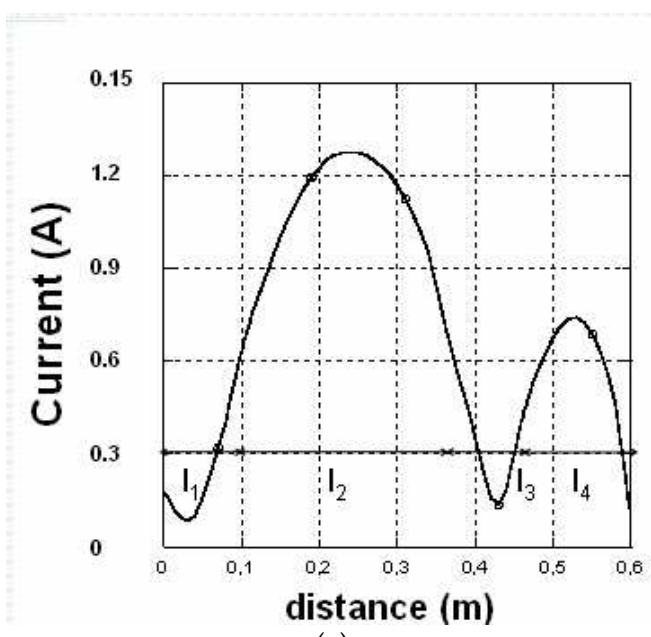

(a)

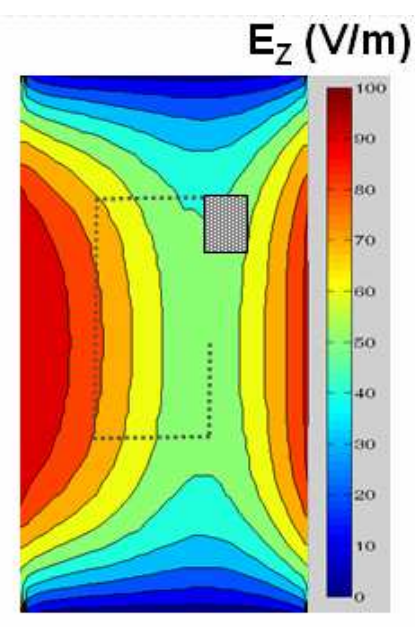

(b)

Fig. 14. Current distribution along the catheter (a), maps of the computed unperturbed electric field in the coronal section passing through the catheter $(b)$

The obtained current distributions can be explained observing that at $64 \mathrm{MHz}$ and in the presence of the dielectric phantom the wavelength is about $50 \mathrm{~cm}$ and hence comparable with the catheter length. Moreover the current inside the wire is mainly produced by the electric field component, obtained in the absence of the wire (unperturbed field in Fig. 14b), parallel to the wire axis. Correspondingly, in the considered exposure condition the strongest currents are produced by the $E_{z}$ field along the $l_{2}$ and $l_{4}$ segments while lower currents are produced by the lower $E_{x}$ component, acting on $l_{1}$ and $l_{3}$.

A further study has been performed in order to investigate the effect of the catheter crosssection on SAR. To this end, two more wires, of radius 0.2 and $0.8 \mathrm{~mm}$, respectively, have been considered. The simulation results, for a circularly polarized magnetic field, have evidenced that the SAR values at the catheter tip increase when the wire section reduces.

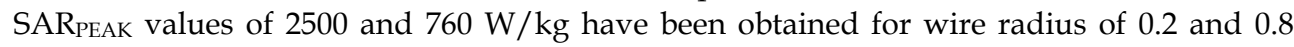
$\mathrm{mm}$, respectively. It is worth noting that the increase of the wire radius determines an 
increase of the current along the wire and at the tip. However, the corresponding increase of the section produces a reduction of the current density and hence the final effect is a reduction of the SAR at the catheter tip.

The effect of the catheter length has been also investigated by considering catheters with a 0.4-mm radius and different lengths. In the considered simulations the distance between the catheter tip and the pacemaker has been maintained equal to $7.5 \mathrm{~cm}$ while the length of the catheter has been changed. Fig. 15 shows the results obtained for a total length of $32 \mathrm{~cm}\left(\mathrm{l}_{1}=\right.$ $\left.7 \mathrm{~cm}, l_{2}=15 \mathrm{~cm}, l_{3}=7 \mathrm{~cm}, l_{4}=3 \mathrm{~cm}\right)$ and for a total length of $44 \mathrm{~cm}\left(l_{1}=10 \mathrm{~cm}, l_{2}=18 \mathrm{~cm}, l_{3}\right.$ $=10 \mathrm{~cm}, 1_{4}=6 \mathrm{~cm}$ ). In these simulations SAR PEAK of 1000 and $1400 \mathrm{~W} / \mathrm{kg}$ have been obtained for the two considered lengths.

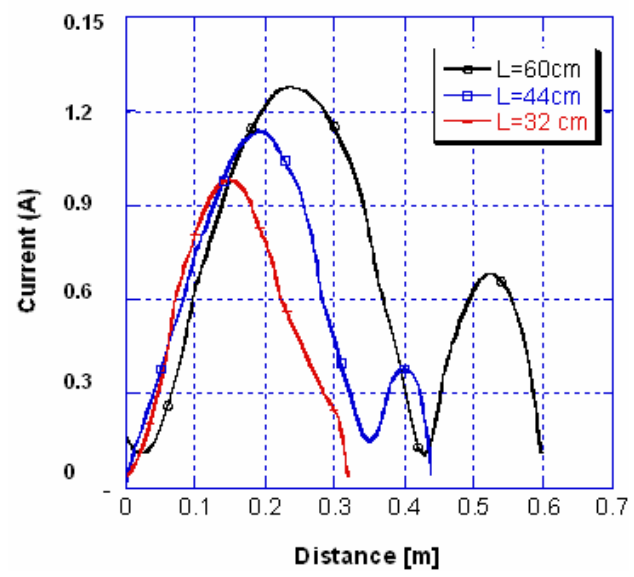

Fig. 15. Current distribution along the catheter for catheters of different lengths

In order to evaluate SAR and temperature distributions in a pacemaker holder in the presence of a more realistic model of the thorax and of the PM, a PM (see Fig. 16a) has been inserted inside the left part of the thorax of the VH model. The obtained model has then been inserted inside the birdcage (see Fig. 12c) and exposed to a $64 \mathrm{MHz}$ left polarized field with a radiated power of $60 \mathrm{~W}$.

Fig. 16b shows the current along the catheter, as a function of the distance from the point in which the catheter is inserted in the metallic box, obtained by considering a plastic covered (PC), a bare (BA), and two uncapped catheters (uncapped for $2 \mathrm{~mm}$ (M2) and uncapped for $6 \mathrm{~mm}(\mathrm{M} 6))$.

The figure shows that for the bare catheter, the current distribution experiences strong variations with the position; these variations, as evidenced in (Pisa et al., 2008), are directly related to the distribution of the electric field parallel to the catheter axis. The presence of the plastic cover makes the current along the catheter smoother; moreover, in the M2 and M6 cases a strong reduction in the current is observed in correspondence of the tip where the catheter is uncapped.

The SAR PEAK $_{\text {at }}$ the catheter tip is $15,700,2400$, and $11900 \mathrm{~W} / \mathrm{kg}$ for the bare, $6 \mathrm{~mm}$ uncapped, $2 \mathrm{~mm}$ uncapped, and plastic covered catheters, respectively. These results show that the PC catheter gives rise to the strongest $S_{A R} R_{P E A K}$ value while the bare catheter 


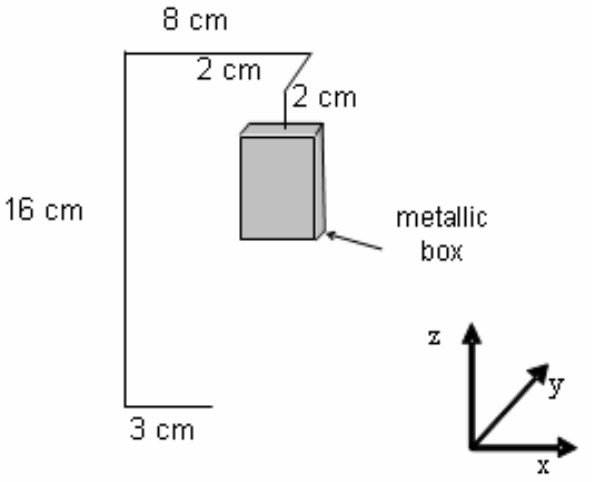

(a)

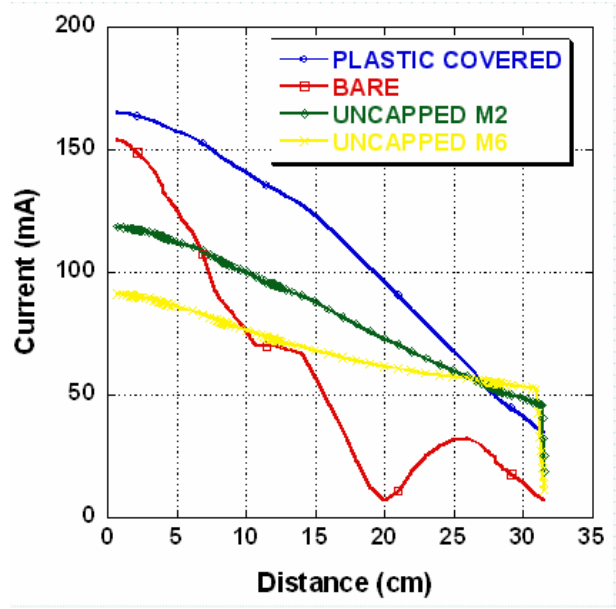

(b)

Fig. 16. Metallic box equipped with a cylindrical catheter (a) and current along the catheter as a function of the distance from the point in which the catheter is inserted in the PM (b) causes the lowest one with a SAR reduction factor of about 800. Intermediate SAR PEAK values are obtained with the two partially capped catheters and the whole set of simulated data is within the range of experimental results reported in literature by considering various PM configurations (Mattei et a., 2008). The $S_{A} R_{10 g}$ values grow from $0.8 \mathrm{~W} / \mathrm{kg}$ for the BA to $9.7 \mathrm{~W} / \mathrm{kg}$ for the PC catheter. Finally, the presence of the PM does not affect significantly the SAR $W B$.

In order to assess the thermal risk associated with the computed SAR values, the graded mesh ADI solution of the bioheat equation has been applied considering the plastic covered catheter that, on the basis of our results, gives rise to the highest SAR. Fig. 17 shows temperature versus time at the catheter tip computed by neglecting the perfusion, in the presence of perfusion, and by adding the convection at the heart-blood interface. In the first case, temperature increments of more than $30^{\circ} \mathrm{C}$ after $10 \mathrm{~min}$ exposure are obtained and the time behavior indicates an exponentially growing trend with a time constant of the order of hours. The presence of blood perfusion reduces temperature increments to $13.5^{\circ} \mathrm{C}$ and, in this case, the steady state is reached in a few minutes. Finally, the presence of convection further reduces temperature increments to less than $13{ }^{\circ} \mathrm{C}$ and $12{ }^{\circ} \mathrm{C}$ for $\mathrm{HB}$ (blood convection coefficient) equal to 100 or $1000 \mathrm{~W} /\left(\mathrm{m}^{2} \cdot{ }^{\circ} \mathrm{C}\right)$, respectively. The results indicate that when blood perfusion is taken into account, a factor of three reduction of temperature increments is obtained with respect to the condition in which only the $\mathrm{C}$ and $\mathrm{K}$ terms are considered. However, the increments are still higher than those indicated as safe in the ICNIRP Statement (2004).

A further result of this study is that a thermal model considering only the $\mathrm{K}, \mathrm{C}, \mathrm{B}$ terms of the $\mathrm{BHE}$ and neglecting $\mathrm{HB}$ allows a worst case estimation of temperature elevations without significantly overestimating the real temperature distributions. Under these conditions, temperature increments of $0.2,1.9$, and $5.8{ }^{\circ} \mathrm{C}$, after $10 \mathrm{~min}$ exposure, are obtained for the BA, UC-M6, and UC-M2 catheters, respectively. 


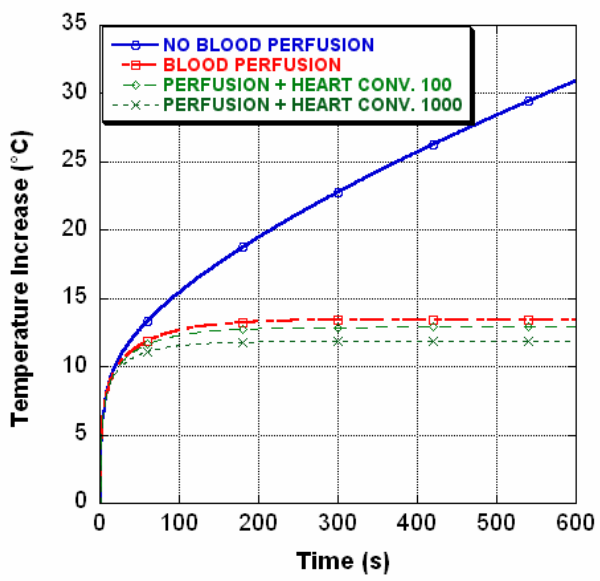

Fig. 17. Temperature increases at the catheter tip computed by neglecting the perfusion, and in the presence of perfusion

\section{Conclusion}

In this chapter the studies available in the literature and concerning SAR distributions and temperature increments in PM holders, exposed to the field generated by MRI apparatus, have been described in order to evidence the main factors influencing the obtained SAR values and temperature increments.

After a preliminary description of MRI apparatus in terms of field sources and distributions, the main in vitro (on phantoms), and numerical literature results have been presented. SAR distributions and temperature increments in PM holders, exposed inside an MRI apparatus, have been evaluated by using an anatomically based human body phantom together with fine pacemaker models equipped with different kinds of catheters, implanted following the vein path. This study has been performed by using a conformal FDTD code with graded mesh for the solution of the electromagnetic (EM) problem, and a finite difference solution of the bioheat equation with graded mesh, that includes blood perfusion in the tissues and convection at the heart-blood and skin-air interfaces, for the evaluation of the corresponding temperature increments.

The principal results of the study carried out are:

- The region with the highest SAR values is the one around the catheter tip where the current flowing along the catheter wire can induce strong current densities in the tissues.

- The current along the catheter is mainly induced by the electric field parallel to the catheter axis. Since strong E-field z-components are present, the highest currents are induced along vertical wires.

- Generally, shorter catheters with shorter vertical wires give rise to lower SAR at the catheter tip. Moreover, SAR values at the catheter tip increase when the wire section reduces. In fact, the increase of the wire radius determines an increase of the current 
along the wire and at the tip. The corresponding increase of the section produces a reduction of the current density and hence the final effect is a reduction of the SAR at the catheter tip.

- The presence of a plastic cover increases the current along the catheter and, consequently, the SAR PEAK at the catheter tip. In particular, the PC catheter gives rise to the strongest SAR PEAK value, while the BA catheter causes the lowest one with an SAR reduction factor of about 800 .

- In the VH model with the PC catheter, temperature increments of more than $30^{\circ} \mathrm{C}$ after $10 \mathrm{~min}$ exposure are obtained and the time behaviour indicates an exponentially growing trend with a time constant of the order of hours. The presence of blood perfusion reduces temperature increments to about $13{ }^{\circ} \mathrm{C}$ and, in this case, the steady state is reached in a few minutes. The presence of convection further reduces temperature increments to less than $12{ }^{\circ} \mathrm{C}$. These results indicate that when blood perfusion is taken into account, a three-fold reduction on temperature increments is obtained with respect to the case in which only the $\mathrm{C}$ and $\mathrm{K}$ terms are considered. However, the increments are still higher than those indicated as safe in ICNIRP (2004).

- In the worst case condition and after 10 min exposure, temperature increments in the $0.2-20^{\circ} \mathrm{C}$ range are obtained on the heart muscle depending on the catheter and body model.

- Closer locations of the PM to the edge of the phantom and to the edge of the coil produce maximum heating.

A further result of this study is that in order to reduce temperature increments below safe levels and hence make MRI possible to PM holders, it is important to reduce the induced currents along the catheter wires. This can be accomplished, for example, by inserting lumped coils along the catheter or by using plastic insulating material with RF losses or, finally, by imaging only parts of the human body far from the PM, such as for example the head and lumbar regions.

\section{References}

Achenbach S., Moshage B., Diem W., Bieberle T., Schibgilla V. \& Bachmann K. (1997), Effects of magnetic resonance imaging on cardiac pacemakers and electrodes, Am. Heart. J., Vol. 134, No. 3, pp. 467-473.

Ackerman M. J. (1998), The Visible Human Project, Proc. IEEE, Vol. 86, pp. 504.

ASTM International Std F218202a (2007), Standard Test Method for Measurement of Radio Frequency Induced Heating Near Passive Implants During Magnetic Resonance Imaging, West Conshohocken, PA, 19428-2959 USA.

Bassen H., Kainz W., Mendoza G. \& Kellom T. (2006), MRI-induced heating of selected thin wire metallic implants - laboratory and computational studies - findings and new questions raised. Minim. Invasive Ther., Vol. 15, No 2, pp. 76-84.

Bernardi P., Cavagnaro M., Pisa S. \& Piuzzi E. (2003), Specific absorption rate and temperature elevation in a subject exposed in the far-field of radio-frequency sources operating in the 10-900-MHz range, IEEE Trans. Biomed. Eng. , Vol. 50, No. 3, pp. 295-304. 
Bernardi P., Cavagnaro M., Pisa S., \& Piuzzi E. (2009), Safety aspects of magnetic resonance imaging for pacemaker holders, in Proceedings of ICEAA '09 (International Conference on Electromagnetics in Advanced Applications), Turin, Italy, pp. 869872 .

Bogdanov G. \& Ludwig R. (2002), Coupled microstrip line transverse electromagnetic resonator model for high-field magnetic resonance imaging, Magn Res in Med Vol. 47, pp. 579-593.

Bridges J.F. (1988), Cavity resonator with improved magnetic field uniformity for high frequency operation and reduced dielectric heating in NMR imaging devices, U.S. Patent 4751464.

Burke J. \& Poggio A. (1981), Numerical electromagnetics code (NEC)-Method of moments, Lawrence Livermore Nat. Lab., Livermore, CA, Tech. Rep. UCID-18834.

Christ A., Kainz W.,. Hahn E.G, Honegger K., Zefferer M., Neufeld E., Rascher W., Janka R., Bautz W., Chen J., Kiefer B., Schmitt P., Hollenbach H.P., Oberle M., Szerba D., Kam A., Guag J.W. \& Kuster N. (2010), The virtual family - development of anatomical CAD models of twoadults and two children for dosimetric simulations, Physics in Medicine and Biology, Vol. 55, pp. 23-38.

Giovannetti G., Landini L., Santarelli M.F. \& Postano V. (2002), A fast and accurate simulator for the design of birdcage coils in MRI. Magn Reson Mat in Phys Biol and Med , Vol. 15, pp. 36-40.

Golombeck M.-A. \& Dossel O. (2004), MR-Tomography on patients with heart pacemakers a numerical study, Proc. 2004 IEEE EMBS Int. Conf., San Francisco USA, pp. 10761079.

Ho H.S. (2001), Safety of metallic implants in magnetic resonance imaging, J. Magn. Reson. Imaging, Vol. 14, No. 4, pp. 472-477.

ICNIRP (2004), Statement on: medical magnetic resonance (MR) procedures: protection of patients, Health Phys., Vol. 87, No 2, pp. 197-216.

Jin J. (1999), Electromagnetic Analysis and Design in Magnetic Resonance Imaging, CRC Press, Boca Raton, FL, USA.

Mason, P.A., Hurt, W.D., Walters, T.J., D’Andrea, J.A., Gajsek, P., Ryan, K.L., Nelson, D.A., Smith, K.I. \& Ziriax, J.M., 2000, Effects of frequency, permittivity, and voxel size on predicted specific absorption rate values in biological tissue during electromagnetic-field exposure, IEEE Trans. Microwave Theory Tech., Vol. 48, ,pp. 2050-2058.

Mattei E., Triventi M., Calcagnini G., Censi F., Kainz W., Bassen H.I. \& Bartolini P. (2007): Temperature and SAR measurement errors in the evaluation of metallic linear structures heating during MRI using fluoroptic probes. Phys. Med. Biol., Vol. 52, No 6, pp. 1633-1646.

Mattei E., Triventi M., Calcagnini G., Censi F., Kainz W., Mendoza G., Bassen H.I. \& Bartolini P. (2008): Complexity of MRI induced heating on metallic leads: Experimental measurements of 374 configurations, BioMedical Engineering OnLine, Vol. 7, No 11. 
Neufeld E., Kuhn S., Szekely G. \& Kuster N. (2009), Measurement, simulation and uncertainty assessment of implant heating during MRI, Phys. Med. Biol., Vol. 54, pp. 4151-4169.

Nyenhuis J. A., Park S.M. \& Kamondetdacha R. (2005), MRI and implanted medical devices: basic interactions with an emphasis on heating, IEEE Trans. Dev. Mat. Reliab., Vol. 5, No 3, pp. 467-480.

Park S.M., Kamondetdacha R., Amjad A. \& Nyenhuis J.A. (2005), MRI safety: RF induced heating on straight wires, IEEE Trans. Magn., Vol. 41, No. 10, pp. 41974199.

Pennes H.H. (1948), Analysis of tissue and arterial blood temperatures in resting forearm, J. Appl. Physiol., Vol. 1, pp. 93-122.

Pisa S., Cavagnaro M., Piuzzi E., Bernardi P. \& Lin J.C. (2003), Power density and temperature distributions produced by interstitial arrays of sleeved-slot antennas for hyperthermic cancer therapy, IEEE Trans. Microwave Theory Tech., vol. 51, No. 12, pp. 2418-2426.

Pisa S, Calcagnini G, Cavagnaro M, Piuzzi E, Triventi M, Bernardi P (2006). SAR and temperature elevations in pacemaker holders exposed to EM fields produced by MRI apparatus. in 2006 IEEE MTT-S International Microwave Symposium Digest, San Francisco: 1754-1757.

Pisa S., Calcagnini G., Cavagnaro M., Piuzzi E., Mattei E. \& Bernardi P. (2008), A study of the interaction between implanted pacemakers and the radio-frequency field produced by magnetic resonance imaging apparatus, IEEE Trans. Electromag. Compat., Vol. 50, No. 1, pp. 35-42.

Pisa S., Bernardi P., Cavagnaro M. \& Piuzzi E. (2010), Power absorption and temperature elevation produced by magnetic resonance apparatus in the thorax of patients with implanted pacemakers, IEEE Trans. Electromag. Compat., Vol. 52, No. 1, pp. 32-40.

Pisa S., Bernardi P., Cavagnaro M. \& Piuzzi E. (2010), Interaction between the RF field of MRI apparatus and pacemaker Holders, Proc. ICEmB Conference, Genova, Italy, pp. 21-22.

Roguin A., Zviman M.M., Meininger G.R., Rodrigues E.R., Dickfeld T.M., Bluemke D.A., Lardo A., Berger R.D., Calkins H. \& Halperin H.R. (2004), Modern pacemaker and implantable cardioverter/defibrillator systems can be magnetic resonance imaging safe. In vitro and in vivo assessment of safety and function at $1.5 \mathrm{~T}$, Circulation, Vol. 110, No. 5, pp. 475-482.

Röschmann P. (1987), Radiofrequency penetration and absorption in the human body: Limitations to high-field whole-body nuclear magnetic resonance imaging. Med Phys, Vol. 14, pp. 922-932.

Röschmann P. (1988), High-frequency coil system for a magnetic resonance imaging apparatus, U.S. Patent 4746866.

Shellock F.G., Fischer L. \& Fieno, S. (2000), Cardiac pacemakers and implantable cardioverter defibrillators: in vitro magnetic resonance imaging evaluation at 1.5Tesla, Journal of Cardiovascular magnetic resonance, Vol. 9, pp. 21-31. 
Sommer T., Vahlhaus C., Lauck G., von Smekal A., Reinke M., Hofer U., Bloch W., Traber F., Schneider C., Gieseke J., Jung W. \& Schild H. (2000), MR imaging and cardiac pacemaker: in-vitro evaluation and in-vivo studies in 51 patients at $0.5 \mathrm{~T}$, Radiology, Vol. 215, No. 3, pp. 869-879.

Stuchly M.A., Abrishamkar H. \& Strydom M.L. (2006), Numerical evaluation of radio frequency power deposition in human models during MRI, Proc. 2006 IEEE EMBS Int. Conf., New York City USA, pp. 272-275.

Taflove A. \& Hagness S.C. (2000), Computational Electrodynamics: The Finite-Difference Time-Domain Method. Boston, MA: Artech House. 


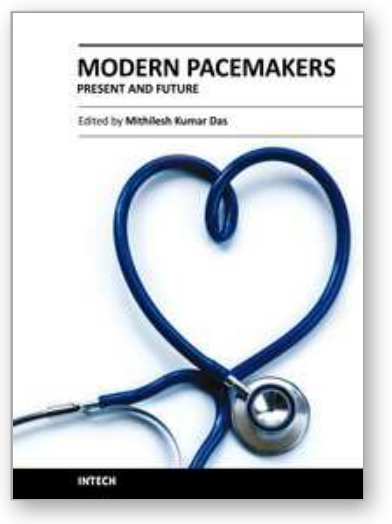

\author{
Modern Pacemakers - Present and Future \\ Edited by Prof. Mithilesh R Das
}

ISBN 978-953-307-214-2

Hard cover, 610 pages

Publisher InTech

Published online 14, February, 2011

Published in print edition February, 2011

The book focuses upon clinical as well as engineering aspects of modern cardiac pacemakers. Modern pacemaker functions, implant techniques, various complications related to implant and complications during follow-up are covered. The issue of interaction between magnetic resonance imaging and pacemakers are well discussed. Chapters are also included discussing the role of pacemakers in congenital and acquired conduction disease. Apart from pacing for bradycardia, the role of pacemakers in cardiac resynchronization therapy has been an important aspect of management of advanced heart failure. The book provides an excellent overview of implantation techniques as well as benefits and limitations of cardiac resynchronization therapy. Pacemaker follow-up with remote monitoring is getting more and more acceptance in clinical practice; therefore, chapters related to various aspects of remote monitoring are also incorporated in the book. The current aspect of cardiac pacemaker physiology and role of cardiac ion channels, as well as the present and future of biopacemakers are included to glimpse into the future management of conductions system diseases. We have also included chapters regarding gut pacemakers as well as pacemaker mechanisms of neural networks. Therefore, the book covers the entire spectrum of modern pacemaker therapy including implant techniques, device related complications, interactions, limitations, and benefits (including the role of pacing role in heart failure), as well as future prospects of cardiac pacing.

\title{
How to reference
}

In order to correctly reference this scholarly work, feel free to copy and paste the following:

Stefano Pisa, Paolo Bernardi and Emanuele Piuzzi (2011). Interaction between the RF Field of MRI Apparatus and Pacemakers, Modern Pacemakers - Present and Future, Prof. Mithilesh R Das (Ed.), ISBN: 978-953-307214-2, InTech, Available from: http://www.intechopen.com/books/modern-pacemakers-present-andfuture/interaction-between-the-rf-field-of-mri-apparatus-and-pacemakers

\section{INTECH}

open science | open minds

\section{InTech Europe}

University Campus STeP Ri

Slavka Krautzeka 83/A

51000 Rijeka, Croatia

Phone: +385 (51) 770447

Fax: +385 (51) 686166

\section{InTech China}

Unit 405, Office Block, Hotel Equatorial Shanghai

No.65, Yan An Road (West), Shanghai, 200040, China 中国上海市延安西路65号上海国际贵都大饭店办公楼 405 单元

Phone: +86-21-62489820

Fax: +86-21-62489821 
www.intechopen.com 
(C) 2011 The Author(s). Licensee IntechOpen. This chapter is distributed under the terms of the Creative Commons Attribution-NonCommercialShareAlike-3.0 License, which permits use, distribution and reproduction for non-commercial purposes, provided the original is properly cited and derivative works building on this content are distributed under the same license. 